\title{
Magnetization-Direction-Dependent Local Electronic Structure Probed by Scanning Tunneling Spectroscopy
}

\author{
M. Bode ${ }^{1, *}$ S. Heinze,${ }^{1,2, \dagger}$ A. Kubetzka, ${ }^{1}$ O. Pietzsch, ${ }^{1}$ X. Nie,${ }^{2}$ G. Bihlmayer, ${ }^{2}$ S. Blügel,${ }^{2, \$}$ and R. Wiesendanger ${ }^{1}$ \\ ${ }^{1}$ Institute of Applied Physics and Microstructure Research Center, University of Hamburg, \\ Jungiusstrasse 11, 20355 Hamburg, Germany \\ ${ }^{2}$ Institut für Festkörperforschung, Forschungszentrum Jülich, 52425 Jülich, Germany
}

(Received 21 December 2001; published 18 November 2002)

\begin{abstract}
Scanning tunneling spectroscopy (STS) of thin Fe films on W(110) shows that the electronic structure of domains and domain walls is different. This experimental result is explained on the basis of first-principles calculations. A detailed analysis reveals that the spin-orbit induced mixing between minority $d_{x y+x z}$ and minority $d_{z^{2}}$ spin states depends on the magnetization direction and changes the local density of states in the vacuum detectable by STS. As a consequence nanometer-scale magnetic structure information is obtained even by using nonmagnetic probe tips.
\end{abstract}

DOI: $10.1103 /$ PhysRevLett.89.237205

PACS numbers: 75.90.+w, 07.79.Cz, 75.60.Ch

Magnetism in reduced dimensions is one of the most active and innovative fields of solid-state physics. Recently, the effect of spin-orbit coupling onto spinpolarized electron wave functions has intensively been investigated as it is the physical basis of magnetic linear [1] and circular [2] x-ray dichroism which - in conjunction with relativistic ab initio calculations-yielded a detailed understanding of the electronic structure of ferromagnets [3]. As an important milestone towards a deeper understanding of magnetism on the nanometer scale, the combination of magnetic circular dichroism with photoemission electron microscopy allowed an imaging of magnetic domain structures at submicrometer resolution [4].

Several authors have analyzed the spin-orbit induced changes in the band structure of thin ferromagnetic films in order to identify the electronic origin of the magnetic anisotropy energy $[5,6]$, the energy difference between the easy and hard axes of the magnetization. It was found theoretically that the electronic structure of ferromagnetic transition metals depends on the magnetization direction. For example, $3 d$-band degeneracies can be lifted by the spin-orbit coupling (SOC) which is magnetization direction dependent [7]. However, the dependence of the electronic structure on the magnetization direction has never been confirmed experimentally by, e.g., angular resolved photoelectron spectroscopy (ARPES), due to (i) the incompatibility of the experimental setup with strong external magnetic fields which are necessary to force the sample magnetization into the hard directions, and (ii) the fact that ARPES can detect only occupied states while significant spin-orbit induced changes are also expected above the Fermi level $\left(E_{\mathrm{F}}\right)$.

We have chosen a different approach: instead of changing the magnetization direction of the whole sample, we make use of the sample's virgin domain state and domain wall structure. In this Letter we show by scanning tunneling spectroscopy (STS) and first-principles calcula- tions that the local surface electronic structure of a $20 \mathrm{~nm}$ stripe of an Fe double layer (DL) on W(110) - a sample with a well-known nanometer-scale domain structure $[8,9]$ - depends on the local orientation of the magnetization. Since the tunneling current of a scanning tunneling microscope (STM), I $\left(\hat{\mathbf{e}}_{M}\left(\mathbf{r}_{\|}\right), \mathbf{r}_{\|}, U\right)$, depends on the electronic structure of the sample, we immediately expect that $I$ is not only a function of the bias voltage $U$ but also depends on the magnetization direction $\hat{\mathbf{e}}_{M}\left(\mathbf{r}_{\|}\right)$of the sample. From a detailed analysis of the electronic structure we deduce how the signature of the magnetization direction is imprinted via the spin-orbit interaction. Surprisingly, we find that the changes in the differential conductivity are on the order of a few percent which is not an obvious consequence of expected band splittings of the order of a few meV. Our analysis reveals that SOC leads to a magnetization direction dependent hybridization of minority bands with different orbital character, thereby changing the density of states above the sample surface. The underlying physics of the spin-orbit dependent differential conductivity can be considered as the static limit of the magnetic dichroism or the tunneling analog of the anisotropic magnetic resistance of ferromagnets. As an important implication of this effect the magnetic nanostructure of surfaces can be investigated with a conventional nonmagnetic tip which has the very advantages that (i) there is definitely no dipolar magnetic interaction between tip and sample which can influence the original magnetic state of the sample and (ii) the preparation of a magnetic tip is omitted.

According to the model of STS by Tersoff and Hamann [10] the differential conductivity

$$
G\left(\mathbf{r}_{\|}, U\right)=\frac{d I}{d U} \mid\left(\mathbf{r}_{\|}, U\right) \propto n\left(\mathbf{r}_{\|}, z, \epsilon=E_{\mathrm{F}}+e U\right),
$$

is proportional to the local density of states (LDOS) $n$ of the sample at the lateral, $\mathbf{r}_{\|}$, and vertical, $z$, position of the tip at the energy $e U$, measured from $E_{\mathrm{F}}$. In case of a 
magnetic sample the wave function is described by a spinor which is diagonalized by a majority $\psi_{\uparrow}|\uparrow\rangle$ and a minority $\psi_{l}|\downarrow\rangle$ spinor if the spin coordinate axis is parallel to the magnetization axis $\hat{\mathbf{e}}_{M}$ and the SOC is omitted. Including SOC in first order perturbation theory, the change in the wave function $\psi_{\mathbf{k}_{\|} \nu}$ of state $\left|\mathbf{k}_{\|} \nu\right\rangle$ is proportional to the expectation value $\left\langle H_{\text {SO }}\right\rangle\left\langle\sigma, \mathbf{k}_{\|} \nu|\mathbf{l s}| \sigma^{\prime} \mathbf{k}_{\|} \nu^{\prime}\right\rangle$ of the spin-orbit Hamiltonian. The orbital part of the matrix element $(\langle\mathbf{I} \mathbf{s}\rangle)$ depends on the magnetization direction and can mix majority and minority states as well as orbitals of the same spin channel, which had been orthogonal without SOC and contribute quite differently to the tunneling current. For example, a slowly dispersing $d_{z^{2}}$ state has a large tunneling cross section, while an in-plane $x y$ state has a small one. Thus we can divide the LDOS into a term $n_{0}$ independent of and a term $\Delta n\left(\hat{\mathbf{e}}_{M}\right)$ dependent on the magnetization direction: $n\left(\hat{\mathbf{e}}_{M}\right)=n_{0}+\Delta n\left(\hat{\mathbf{e}}_{M}\right)$. For a system with uniaxial out-of-plane anisotropy $\Delta n\left(\hat{\mathbf{e}}_{M}\right) \propto$ $\sin ^{2} \theta$, where $\theta$ is the angle between the magnetization direction and the surface normal.

The experiments on different $1.75 \pm 0.1 \mathrm{ML}$ thick Fe films on $\mathrm{W}(110)$ have been performed in an ultrahigh vacuum system with separate chambers for substrate preparation, sample transfer, metal vapor deposition, surface analysis, and cryogenic STM [11]. The base pressure in each chamber is in the low $10^{-11}$ torr range. We used etched polycrystalline $\mathrm{W}$ tips which were flashed in vacuo at $T>2200 \mathrm{~K}$. During the measurements, tip and sample were at a temperature $T=15 \mathrm{~K}$.

Fe grows in the step-flow mode; i.e., the W substrate's step edges are decorated with Fe nanostripes of alternating monolayer and double layer thickness $[8,9,12]$ [Fig. 1(a)]. The spin-polarized STS images of Figs. 1(b) and 1 (c) reveal that the sample exhibits perpendicularly magnetized stripe domains running along the [110] direction with a lateral magnetic periodicity of $\approx 50 \mathrm{~nm}$ : while the magnetic domain structure is imaged with the out-of-plane sensitive Gd tip, the Fe coated tip is sensitive to the in-plane magnetization [13] and opposite in-plane magnetization directions ([11 10$]$ and [ 110$])$ lead to opposite contrasts. As can be recognized in Fig. 1(c), our results show that the magnetization direction alternatingly changes between both directions [9]. Surprisingly, the domain walls are also visible in data which have been measured with a nonmagnetic W tip [Fig. 1(d)]. However, all domain walls exhibit the same contrast; i.e., at the position of the domain wall the differential conductivity $d I / d U$ is reduced with respect to the domain. As revealed by the local tunneling spectra [Fig. 1(e)], this contrast is caused by a tiny difference which is energetically located just above $E_{\mathrm{F}}$ (see inset): while the $d I / d U$ spectrum measured with the tip positioned above the domain exhibits a weak peak at $U=0.07 \mathrm{~V}$, this peak is almost absent in the domain wall spectra. This is further illustrated by the plot of the normalized difference $A\left(\mathbf{r}_{\|}, U\right)=$ $\left[G\left(\mathbf{r}_{\|}, U\right)-G\left(\overline{\mathbf{r}}_{\|}, U\right)\right] /\left[G\left(\mathbf{r}_{\|}, U\right)+G\left(\overline{\mathbf{r}}_{\|}, U\right)\right]\left[\mathbf{r}_{\|}\left(\overline{\mathbf{r}}_{\|}\right):\right.$position in a domain (domain wall)] in the lower panel of

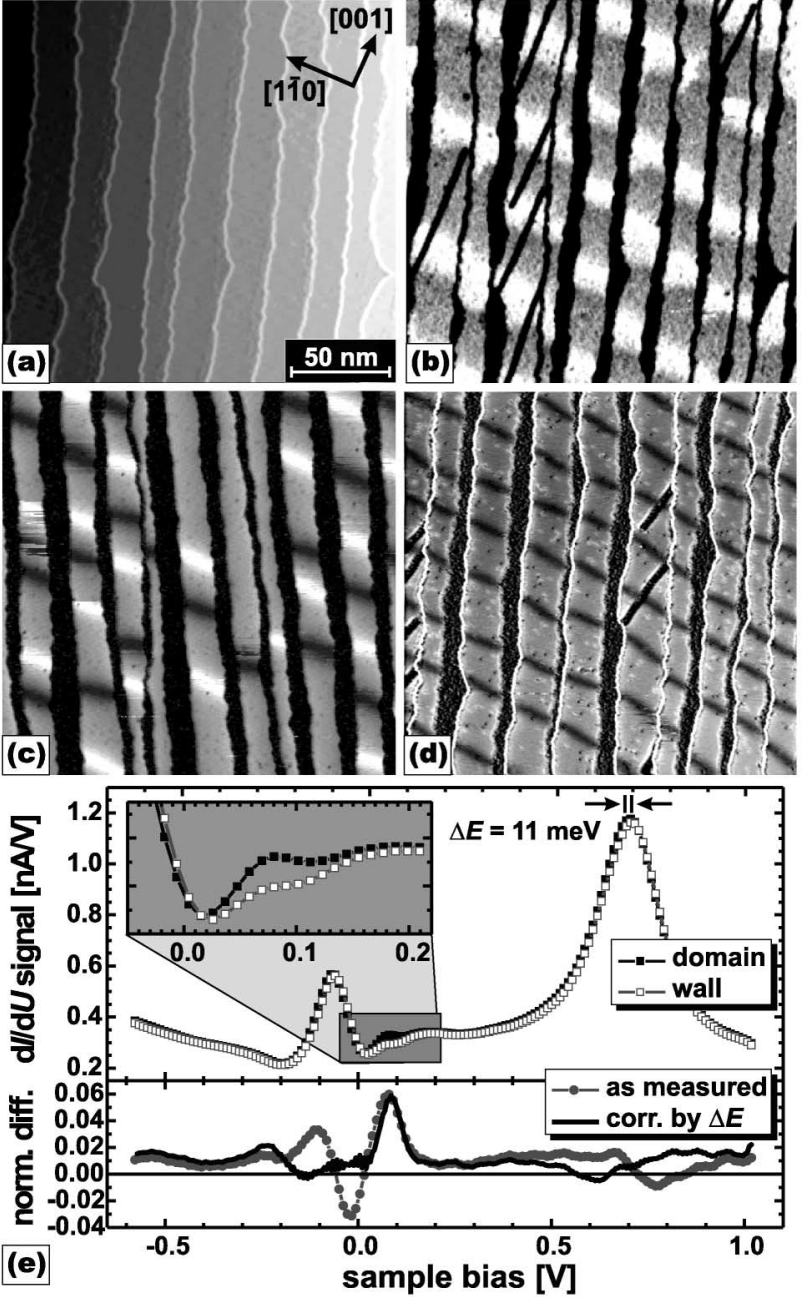

FIG. 1. (a) Topography and magnetic $d I / d U$ signal of $1.75 \pm$ $0.1 \mathrm{ML} \mathrm{Fe} / \mathrm{W}(110)$ as measured with (b) a Gd coated $(U=$ $0.7 \mathrm{~V}),(\mathrm{c})$ a $\mathrm{Fe}$ coated $(U=0.7 \mathrm{~V})$, and $(\mathrm{d})$ an uncoated $\mathrm{W}$ probe tip $(U=0.05 \mathrm{~V})$. (e) $d I / d U$ spectra of domains and domain walls.

Fig. 1(e). However, if the normalized difference is calculated on the original data (as measured), a pronounced oscillation can be found just below $E_{\mathrm{F}}$, i.e., at small negative sample bias. This oscillation is not caused by any additional or missing spectroscopic features in the domain wall $d I / d U$ spectrum with respect to the spectrum measured at domains but by an overall energetic shift $\Delta E=11 \mathrm{meV}$. The physical origin is different work functions in domains and domain walls as already proposed in Refs. [14,15]. It can be corrected by shifting the domain wall spectrum by $-\Delta E$. After this procedure, the oscillation below $E_{\mathrm{F}}$ has almost perfectly disappeared.

To interpret the experiments we have performed firstprinciples calculations based on the density functional theory (DFT). Using our FLEUR code [16] which is an implementation of the full-potential linearized augmented plane wave method in the film geometry [17] including SOC second variational procedure, we have calculated the electronic structure of $2 \mathrm{ML} \mathrm{Fe} / \mathrm{W}(110)$ 
as a function of the magnetization direction. The films consisted of five layers of $\mathrm{W}$ and two layers of $\mathrm{Fe}$ on each side of the film. For the structural model we used the experimental W lattice constant $\left(a_{0}=3.167 \AA\right)$ and relaxed the Fe layers by force calculations applying the generalized gradient approximation [18]. Electronic structure calculations including spin-orbit interactions were carried out using the local density approximation [19]. The structural optimization to determine the equilibrium interlayer distances was carried out neglecting the spin-orbit interaction. The 33 special $\mathbf{k}_{\|}$points in the irreducible wedge of the two-dimensional Brillouin zone (I-2D-BZ) $(1 / 4$ of BZ) of the chemical unit cell were used. The spin-orbit dependent contributions to the tunneling current are carried out with a much larger set of $\mathbf{k}_{\|}$ points (see figure captions) in the magnetic I-2D-BZ ( $1 / 2$ of BZ).

Figure 2 shows the vacuum LDOS, $n(E)$, for two different magnetization directions of Fe: out-of-plane, representing the magnetization state (full line) in the domain, and in-plane along the [110] direction (dashed line) as a model of the magnetization in the domain wall. At first glance, the results seem indistinguishable. We find two $d_{z^{2}}$ states at the $\mathrm{Fe}$ surface leading to pronounced peaks at -0.18 and $+0.85 \mathrm{eV}$, characteristic for the Fe DL on W. Since these states are located at the $\bar{\Gamma}$ point of the $2 \mathrm{D}-\mathrm{BZ}$, they should be easily detected by STS [20]. In fact, they can be identified with the peaks at -0.08 and $+0.7 \mathrm{~V}$ in the experimental spectra. A spin analysis reveals that these are basically of minority character (neglecting the small spin mixing due to the spin-orbit interaction). A closer look (cf. inset in Fig. 2) reveals a significant enhancement of the LDOS for the out-of-plane magnetized film within an energy interval of about $100 \mathrm{meV}$ above $E_{\mathrm{F}}$. The lower panel of Fig. 2 shows the normalized

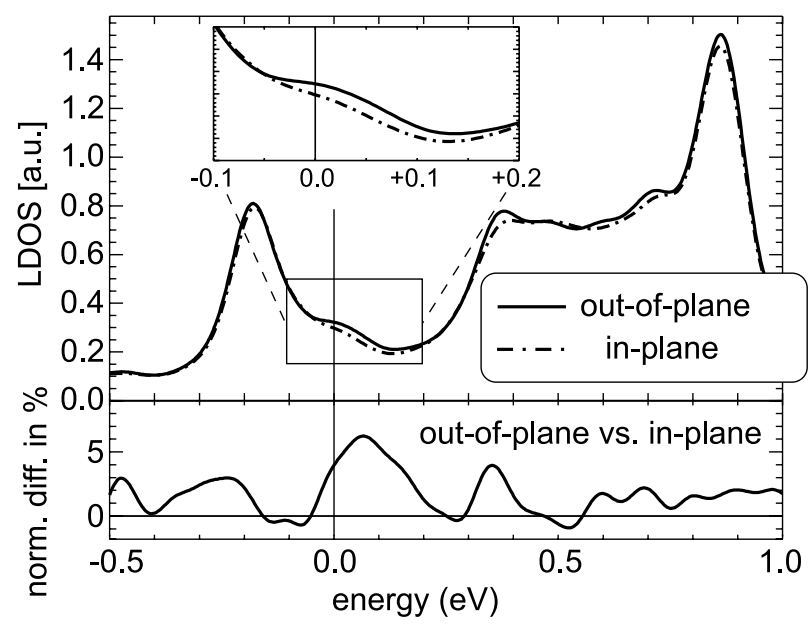

FIG. 2. Calculated LDOS at about $z=14 \AA$ above the surface of $2 \mathrm{ML} \mathrm{Fe} / \mathrm{W}(110)$, measured from the center of the atom. The lower panel displays the normalized difference. For the calculation $3600 \mathbf{k}_{\|}$points have been used in a square centered at $\bar{\Gamma}$ with an area covering $25 \%$ of the BZ (see Fig. 3 ). difference. Both the peak in the normalized difference and the enhancement of the LDOS right above $E_{\mathrm{F}}$ are in very nice accordance with the experimental results of Fig. 1(e). The peak at $-0.3 \mathrm{eV}$ in the normalized difference may correspond to the peak at $-0.24 \mathrm{eV}$ in Fig. 1(e), while the peak at $0.35 \mathrm{eV}$ cannot be found in the experimental data.

In reality, within the domain wall which is about $6 \mathrm{~nm}$ wide [8], the magnetization rotates gradually from the out-of-plane to the in-plane direction. Thus, we have carried out further calculations for magnetizations tilted with respect to the out-of-plane direction by $\theta=22.5^{\circ}$, $45^{\circ}$, and $67.5^{\circ}$. While the reduction in the LDOS above $E_{\mathrm{F}}$ is absent for $\theta=22.5^{\circ}$, it appears for $\theta=45^{\circ}$ and $67.5^{\circ}$ which explains - compared to the result obtained with an Fe coated tip - the apparently reduced domain wall width as measured with the $\mathrm{W}$ tip [cf. Figures 1(c) and $1(\mathrm{~d})$ ]. We have further confirmed that the experimentally observed effect is absent when the magnetization is oriented in-plane along the [001] direction.

The $\mathbf{k}_{\|}$-resolved contributions over the $2 \mathrm{D}-\mathrm{BZ}$ of the minority projected part of the LDOS at an energy of $E_{\mathrm{F}}+$ $50 \mathrm{meV}$ are plotted in Fig. 3(a) for the out-of-plane direction. All states have been smeared by a Gaussian of $50 \mathrm{meV}$. We identify a spot at the $\bar{\Gamma}$ point and two stretched spots at the inner circle in the $\overline{\Gamma P}$ direction as the origin of the vacuum LDOS at this energy. In the difference plot between the LDOS of out-of-plane and inplane magnetization presented in Fig. 3(b) we observe that the states at the inner circle cause the changes.

In order to understand the nature of the changes of the electronic structure upon rotation of the magnetization, the band structure has been plotted in Fig. 4 along the line $\overline{\Gamma P}$ drawn in Fig. 3. In Fig. 4(a) the spin-orbit interaction is turned off and majority and minority states diagonalize
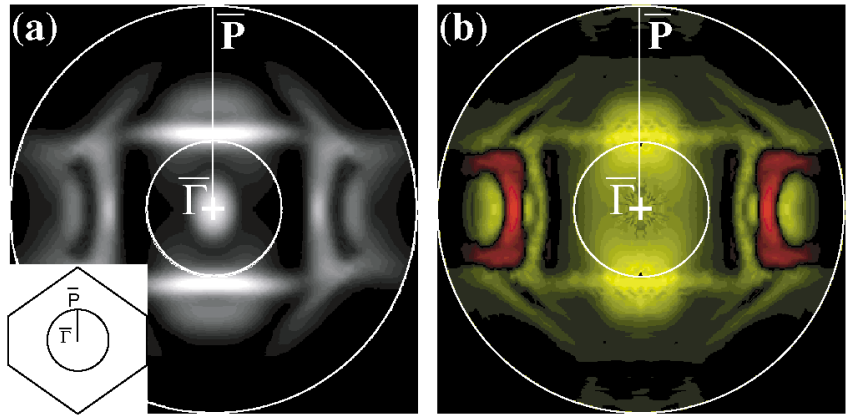

FIG. 3 (color). $\mathbf{k}_{\|}$-resolved contributions to the minority LDOS for states with an energy of $E_{\mathrm{F}}+50 \mathrm{meV}$ displayed in the $2 \mathrm{D}-\mathrm{BZ}$. A disk of radius $\overline{\Gamma \mathrm{P}}$ centered around the $\bar{\Gamma}$ point with an area of $20 \%$ of the $2 \mathrm{D}-\mathrm{BZ}$ has been chosen. States with larger $\left|\mathbf{k}_{\|}\right|$contribute only insignificantly to the vacuum LDOS. (a) The LDOS for the out-of-plane direction, and (b) the difference $\Delta n$ between the LDOS $n$ for out-of-plane and in-plane magnetization directions. Yellow (red) corresponds to positive (negative) values of $\Delta n$. A total of 6000 $\mathbf{k}_{\|}$points have been used to sample the disk. 


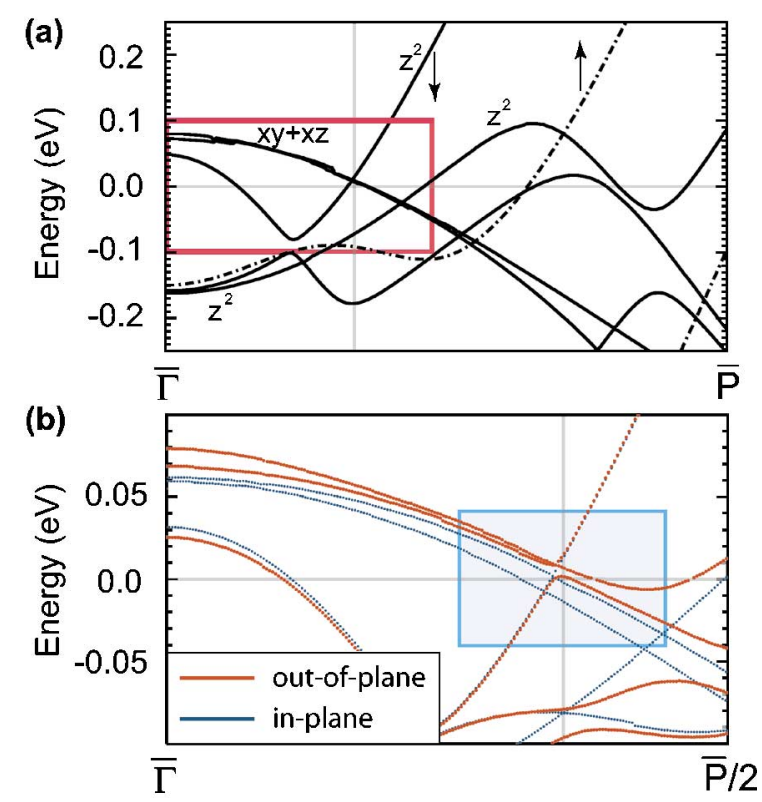

FIG. 4 (color). Comparison of the band structure of the entire Fe DL film along the line $\overline{\Gamma P}$ (cf. Fig. 3) (a) without and (b) with spin-orbit interaction. In (a) the full (chained) line represents the minority (majority) bands. The view of (b) is shown as a red box in (a). The vertical grey lines indicate the radius of the inner circle of Fig. 3. A total of $400 \mathbf{k}_{\|}$points along $\overline{\Gamma P}$ have been used to plot the band structure.

the Hamiltonian. We find that in the $\mathbf{k}_{\|}$region of interest three minority state bands cross at $E_{\mathrm{F}}$. One is rising while two nearly degenerate ones are falling. Note that the contribution of a state to the differential conductivity $G$ or the vacuum LDOS $n$, respectively, is weighted by its inverse group velocity $\left(d E / d \mathbf{k}_{\|}\right)^{-1}$, the relative position with respect to the $\bar{\Gamma}$ point $\left[\propto \exp \left(-\alpha k_{\|}^{2} z\right)\right]$, and depends on its orbital character. Therefore, the $d_{z^{2}}$ state at the $\bar{\Gamma}$ point dominates the vacuum LDOS and causes, e.g., a peak in the vacuum LDOS at $-0.18 \mathrm{eV}$ (see Fig. 2). On the other hand, the states with the $d_{x y}$ and $d_{x z}$ orbital character or the rapidly dispersing $d_{z^{2}}$ band for states off the $\bar{\Gamma}$ point as shown in Fig. 4(a) contribute only by a negligible amount. However, depending on the magnetization direction, the SOC can mix the three crossing bands. As shown in the center of the blue box in Fig. 4(b) this happens actually for the out-of-plane magnetization, which leads to an hybridization gap between one of the $d_{x y+x z}$ and the $d_{z^{2}}$ states. The introduction of this gap changes the group velocity of the $d_{z^{2}}$ state upon changing the magnetization direction, which explains the effect detected by STS.

In summary, we have observed by STS that the electronic structure of a thin Fe film on W(110) depends on the magnetization direction. First-principles calculations based on the DFT reveal that the observed difference is caused by spin-orbit coupling which leads to a magnetization direction dependent hybridization gap between the minority $d_{x y+x z}$ and $d_{z^{2}}$ spin states, causing a magnetiza- tion direction dependent contribution to the tunneling current. The effect has been observed on $2 \mathrm{ML} \mathrm{Fe/}$ $\mathrm{W}(110)$ using many different tip materials. Since the magnetization dependent term of the LDOS $\Delta n\left(\hat{\mathbf{e}}_{M}\right) \propto$ $\sin ^{2} \theta$, it is believed that the effect is even visible on samples with rather broad domain walls. Furthermore, our theoretical results indicate that similar effects can also be found on Fe films on $\mathrm{Mo}(110)$, a system which exhibits a much smaller SOC. Therefore, we expect that the effect should be quite general and applicable to a large variety of systems.

We acknowledge financial support from the BMBF (Grant No. 13N7647) and the DFG (Grant No. Wi 1277/ 16 and Graduiertenkolleg "Physik nanostrukturierter Festkörper"). We are grateful to K. Starke (Berlin) for insightful discussions.

*Email address: mbode@ physnet.uni-hamburg.de ${ }^{\dagger}$ Present address: IBM Research Division, T. J. Watson Research Center, Yorktown Heights, NY 10598.

‡Permanent address: Fachbereich Physik, Universität Osnabrück, 49069 Osnabrück, Germany.

[1] G. van der Laan et al., Phys. Rev. B 34, 6529 (1986).

[2] G. Schütz et al., Phys. Rev. Lett. 58, 737 (1987).

[3] W. Kuch and C. M. Schneider, Rep. Prog. Phys. 64, 147 (2001).

[4] G. Schönhense, J. Phys. Condens. Matter 11, 9517 (1999).

[5] G. H. O. Daalderop, P. J. Kelly, and M. F. H. Schuurmans, in Ultrathin Magnetic Structures I, edited by J. A.C. Bland and B. Heinrich (Springer, Berlin, 1994).

[6] R. Wu and A. J. Freeman, J. Magn. Magn. Mater. 200, 498 (1999).

[7] A. Lessard, T. H. Moos, and W. Hübner, Phys. Rev. B 56, 2594 (1997).

[8] O. Pietzsch et al., Phys. Rev. Lett. 84, 5212 (2000).

[9] M. Bode et al., Phys. Rev. Lett. 86, 2142 (2001).

[10] J. Tersoff and D. R. Hamann, Phys. Rev. Lett. 50, 1998 (1983).

[11] O. Pietzsch et al., Rev. Sci. Instrum. 71, 424 (2000).

[12] H. J. Elmers, J. Hauschild, and U. Gradmann, Phys. Rev. B 59, 3688 (1999).

[13] Here the discussion of magnetic properties is restricted to the Fe DL. Because of its different electronic properties [8] the Fe monolayer appears black at the selected values of the sample bias [see Figs. 1(b)-1(d)].

[14] G. H. O. Daalderop, P. J. Kelly, and M. F. H. Schuurmans, Phys. Rev. B 50, 9989 (1994).

[15] M. I. Darby and E. D. Isaac, IEEE Trans. Magn. 10, 259 (1974).

[16] http://www.flapw.de

[17] E. Wimmer et al., Phys. Rev. B 24, 864 (1981); M. Weinert, E. Wimmer, and A. J. Freeman, ibid. 26, 4571 (1982).

[18] J. P. Perdew et al., Phys. Rev. B 46, 6671 (1992).

[19] V. L. Moruzzi, J. F. Janak, and A. R. Williams, Calculated Electronic Properties of Metals (Pergamon, New York, 1978).

[20] J. A. Stroscio et al., Phys. Rev. Lett. 75, 2960 (1995). 